12

\title{
Плазмон-экситонные поляритоны в сверхрешетках
}

\author{
(C) В.А. Кособукин \\ Физико-технический институт им. А.Ф. Иофрфе РАН, \\ Санкт-Петербург, Россия \\ E-mail: Vladimir.Kosobukin@mail.ioffe.ru
}

(Поступила в Редакцию 3 октября 2016 г.)

\begin{abstract}
Представлена теория распространения поляритонов в сверхрешетках с резонансным плазмон-экситонным взаимодействием. Периодическая сверхрешетка состоит из конечного числа ячеек с близко расположенными квантовой ямой и монослоем металлических наночастиц. Изучается спектр смешанных мод, образованных квазидвумерными экситонами квантовых ям и дипольными плазмонами металлических частиц. Задача электродинамики решается методом функций Грина с учетом резонансной поляризации квантовых ям и частиц в самосогласованном приближении. Эффективная поляризуемость частиц сфероидальной формы, заполняющих квадратную решетку, вычислена с учетом эффекта локального поля дипольных плазмонов слоя и их „изображений“, обусловленных экситонной поляризацией ближайшей квантовой ямы. Численно исследованы оптические спектры отражения сверхрешеток с квантовыми ямами GaAs/AlGaAs и частицами серебра. Особое внимание уделено режиму сверхизлучения при брэгговской дифракции поляритонов в сверхрешетке. Сверхизлучение исследовано для плазмонов и экситонов по отдельности, а также для смешанных плазмон-экситонных поляритонов. Показано, что широкий спектр отражения, связанный с плазмонами, зависит от числа ячеек в сверхрешетке и имеет узкий спектральный провал в области экситонплазмонного расщепления Раби.
\end{abstract}

Работа выполнена при частичной поддержке РФФИ (грант № 14-02-01123).

DOI: $10.21883 /$ FTT.2017.05.44389.365

\section{1. Введение}

Низкоразмерные экситоны и локализованные плазмоны представляют собой разновидности коллективных электронных возбуждений со спектром в видимом диапазоне. Они имеют существенно разные оптические свойства: экситонные резонансы в полупроводниках узкие, а поверхностные плазмоны в металлах имеют сравнительно большую ширину при значительной добротности. Индивидуальные свойства поляризационных возбуждений обоих типов достаточно хорошо известны $[1,2]$ и находят разнообразные практические приложения благодаря возможности управлять ими. Актуальным становится изучение резонансных оптических явлений, обусловленных кулоновским взаимодействием плазмонов с низкоразмерными экситонами в наноструктурах металл-полупроводник $[3,4]$.

Установлено, что взаимодействие экситонов и плазмонов может приводить к возмущению их состояний [5] или к образованию смешанных состояний [6-11]. Под влиянием плазмонов меняется скорость спонтанного распада экситонов [12-14], появляется возможность концентрации электромагнитной энергии на нанометровом масштабе и ее переноса в отсутствие переноса носителей заряда [12] и т.д. При сильном взаимодействии экситонов с плазмонами в спектрах молекулярных систем [6-9] и квантовых ям [10] обнаружено экситон-плазмонное расщепление Раби большой величины. Влияние плазмонов на экситоны большого радиуса исследовалось преимущественно для нульмерных экситонов в квантовых точках или микрокристал- лах [9,11-14] и для квазидвумерных экситонов в квантовых ямах [10,15-17] или полупроводниковых покрытиях [18]. В работах $[19,20]$ изучались также оптические переходы в квантовых ямах с участием электромагнитных мод массива наноантенн. В работе [16] была развита теория упругого рассеяния света в области плазмонэкситонного резонанса металлической наночастицы, расположенной вблизи от квантовой ямы. Эффекты взаимодействия плазмонов и экситонов, возбуждаемых светом в близко расположенных слое наночастиц металла и квантовой яме, теоретически изучались и в отражении света [17]. В оптических спектрах рассеяния и отражения было предсказано значительное расщепление Раби в гибридной плазмон-экситонной моде.

Остается открытым важный вопрос о формировании поляритонов в сверхрешетках металл-полупроводник при взаимодействии плазмонов с экситонами. В этом случае особый интерес представляет возможность брэгговской дифракции плазмон-экситонных поляритонов, которая ожидаемо должна приводить к сверхизлучению, как в полупроводниковых сверхрешетках [21]. Для последних экситонные поляритоны в режиме сверхизлучения изучались при нормальном [22,23] и наклонном [24] падении, включая случай, когда ячейки сверхрешетки содержат более одной квантовой ямы [23].

В данной работе решается задача о распространении электромагнитных волн (поляритонов) в сверхрешетках в спектральной области плазмон-экситонных резонансов. Рассмотрение относится к периодической последовательности сверхъячеек, включающих близко расположенные квантовую яму и слой частиц с лока- 
лизованными плазмонами. Внутри таких ячеек взаимодействие экситонов и плазмонов осуществляется через их ближнее (кулоновское) поле. Взаимодействие между ячейками через запаздывающее электромагнитное поле имеет интерференционный характер. Теория развита на основе метода многократного резонансного рассеяния света в наноструктурах металл-полупроводник при наличии локальных плазмонов и квазидвумерных экситонов. Получено самосогласованное решение задачи о вычислении плазмон-экситонного отклика с учетом эффекта локального поля. Содержание статьи заключается в следующем. В разд. 2 обсуждается плазмонэкситонная модель сверхрешетки, в разд. 3 для нее получено общее решение электродинамической задачи. Матрицы переноса вычислены в разд. 4. В разд. 5 приводятся результаты численного расчета плазмонэкситонных спектров отражения, преимущественно в режиме сверхизлучения.

\section{2. Модель}

Рассмотрим периодическую сверхрешетку, состоящую из $N$ ячеек в однородном оптически изотропном полупроводнике с проницаемостью $\varepsilon_{b}$. Модель сверхрешетки схематически показана на рис. 1. Идентичные ячейки образованы близко расположенными друг к другу слоем металлических наночастиц (NP) и квантовой ямой $(\mathrm{QW})$; для краткости эту пару далее будем называть бислоем (BL). Резонансная дипольная поляризация сверхрешетки $\mathbf{P}=\mathbf{P}^{(\mathrm{I})}+\mathbf{P}^{(\mathrm{II})}$, индуцированная световым полем, состоит из вклада $\mathbf{P}^{(\mathrm{I})}$ квазидвумерных экситонов квантовых ям и вклада $\mathbf{P}^{(\mathrm{II})}$ локализованных плазмонов наночастиц. Считаем, что период сверхрешетки равен $d$, центр ее $n$-й ячейки имеет координату $z=z_{0, n}=(n-1 / 2) d$, а слой наночастиц и квантовая яма в ней центрированы при $z=z_{p, n}=z_{0, n}-h / 2$ и $z=z_{e, n}=z_{0, n}+h / 2$ соответственно, где индексы $p$ и $e$ относятся к плазмонам и экситонам. Ширина квантовой ямы, размеры металлических частиц и их удаление $h=z_{e, n}-z_{p, n}>0$ от квантовой ямы в ячейке малы по сравнению с длиной световой волны $2 \pi /\left(\sqrt{\varepsilon_{b}} k_{0}\right)$, где $k_{0}=\omega / c, \omega-$ частота, $c-$ скорость света. Как следствие, взаимодействие между экситонами и плазмонами в ячейке имеет кулоновский характер.

Следуя $[1,25,26]$, экситонную поляризацию квантовых ям представляем в виде

$$
\begin{gathered}
P_{\alpha}^{(\mathrm{I})}(z, \boldsymbol{\rho})=\frac{1}{4 \pi} \int \Delta \varepsilon^{(0)}\left(z, z^{\prime}\right) E_{\alpha}\left(z^{\prime}, \boldsymbol{\rho}\right) d z^{\prime} \\
\frac{1}{4 \pi} \Delta \varepsilon^{(0)}\left(z, z^{\prime}\right)=\sum_{n} \psi\left(z-z_{e, n}\right) \frac{\Gamma_{0}}{\omega_{0}-\omega-i \Gamma} \psi\left(z^{\prime}-z_{e, n}\right) .
\end{gathered}
$$

Здесь $\omega_{0}$ - частота экситона, $\Gamma_{0}$ и $Г-$ параметры экситон-фотонного взаимодействия и диссипативного затухания экситона соответственно, $\boldsymbol{\rho}=(x, y)$. Огибающая волновой функции основного экситонного состояния $\psi(z)=\psi(-z)$ отлична от нуля в квантовой

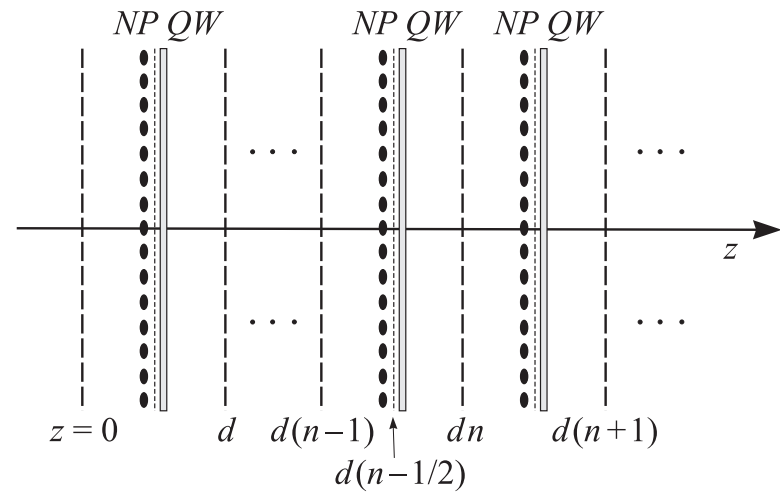

Рис. 1. Схематическое изображение сверхрешетки с периодом $d$, в каждой ячейке которой находится бислой (BL), образованный близко расположенными слоем наночастиц (NP) и квантовой ямой $(\mathrm{QW})$.

яме шириной $l$, при этом $\int d z \psi(z)=\sqrt{l}, \int d z \psi^{2}(z)=1$. Экситоннные волновые функции разных ям не перекрываются: $\psi\left(z-z_{e, n}\right) \psi\left(z-z_{e, n^{\prime}}\right)=\delta_{n n^{\prime}} \psi^{2}\left(z-z_{e, n}\right)$.

Энергия возбуждения экситонов в полупроводниках $\hbar \omega_{0} \approx 1.5 \mathrm{eV}$ (для GaAs), а энергия дипольных поверхностных плазмонов в металлических шаровых частицах составляет несколько электрон-вольт. Однако путем трансформации формы частиц в эллипсоидальную и изменения расстояний между частицами энергию плазмонов можно уменьшить до резонанса с экситонами. Для слоя эллипсоидальных частиц поляризация дипольных плазмонов равна $[27,28]$

$P_{\alpha}^{(\mathrm{II})}(z, \boldsymbol{\rho})=\sum_{\mathbf{m}, n} \chi_{\alpha \alpha}^{(0)}(\omega) \delta\left(z-z_{p, n}\right) \delta\left(\boldsymbol{\rho}-\boldsymbol{\rho}_{\mathbf{m}}\right) E_{\alpha}\left(z_{p, n}, \boldsymbol{\rho}_{\mathbf{m}}\right)$,

где дельта-функция $\delta\left(z-z_{p, n}\right) \delta\left(\boldsymbol{\rho}-\boldsymbol{\rho}_{\mathbf{m}}\right)$ означает, что в слое $z=z_{p, n}$ центры частиц находятся в точках $\boldsymbol{\rho}=\boldsymbol{\rho}_{\mathbf{m}}$. Далее мы рассматриваем частицы в форме эллипсоидов вращения (сфероидов), сжатых вдоль оси сверхрешетки. В главных осях сфероида компоненты тензора поляризуемости $\hat{\chi}^{(0)}$ изолированной частицы выражаются формулой [29]

$$
\chi_{\alpha \alpha}^{(0)}=\frac{a^{3} \eta}{3} \frac{\varepsilon-\varepsilon_{b}}{\left(\varepsilon-\varepsilon_{b}\right) N_{\alpha}+\varepsilon_{b}} .
$$

Здесь $\varepsilon$ и $\varepsilon_{b}$ - диэлектрические проницаемости материала частицы и однородной окружающей среды, $N_{\alpha}$ - коэффициент деполяризации, зависящий от относительной длины $\eta=b / a$ полуосей $a_{x}=a_{y}=a$ и $a_{z}=b$ сфероида.

Для металлических частиц $\varepsilon(\omega)=\varepsilon_{\infty}-\omega_{p}^{2} /\left(\omega^{2}+i \omega \gamma\right)$ в (4), где $\omega_{p}$ - частота плазменных колебаний электронного газа, $\gamma$ - параметр затухания (обратное время релаксации электронов). Выражение (4) преобразуется к виду $[27,28]$

$$
\chi_{\alpha \alpha}^{(0)}=\frac{a^{3} \eta}{3} \frac{\left(\Omega_{\alpha}^{(0)}\right)^{2}}{\left(\omega_{\alpha}^{(0)}\right)^{2}-\omega^{2}-i \omega \gamma} .
$$


В этой формуле $\omega_{\alpha}^{(0)}=\omega_{p}\left(N_{\alpha} / \varepsilon_{*}^{(\alpha)}\right)^{1 / 2}-$ частота плазмона с поляризацией вдоль $\alpha$-й оси в изолированном сфероиде, $\Omega_{\alpha}^{(0)}=\omega_{\alpha}^{(0)} \sqrt{\varepsilon_{b} /\left(N_{\alpha} \varepsilon_{*}^{(\alpha)}\right)}$, где $\varepsilon_{*}^{(\alpha)}=\left(\varepsilon_{\infty}-\varepsilon_{b}\right) N_{\alpha}+\varepsilon_{b}$. В выражении (5) мы пренебрегаем радиационными поправками: затуханием $\sim \omega_{\alpha}^{(0)}\left(\omega_{\alpha}^{(0)} a / c\right)^{3}$, малым по сравнению с $\gamma$, а также сдвигом $\sim \omega_{\alpha}^{(0)}\left(\omega_{\alpha}^{(0)} a / c\right)^{2}$ частоты $\omega_{\alpha}^{(0)}$ (оценки относятся к плазмону малой $\left(a \ll c / \omega_{\alpha}^{(0)}\right)$ шаровой частицы [30,31]).

\section{3. Общее решение электродинамической задачи}

Рассмотрим задачу электродинамики при наличии поляризации $\mathbf{P}=\mathbf{P}^{(\mathrm{I})}+\mathbf{P}^{(\mathrm{II})}$ с вкладами (1) и (3). Используем уравнение для поля

$$
\left(\operatorname{rot} \operatorname{rot}-\varepsilon_{b} k_{0}^{2} \hat{I}\right) \mathbf{E}=4 \pi k_{0}^{2}\left(\mathbf{P}^{(\mathrm{I})}+\mathbf{P}^{(\mathrm{II})}\right),
$$

где $\hat{I}$ - единичный тензор с компонентами $\delta_{\alpha \alpha}=1$ при $\alpha=\beta$ и $\delta_{\alpha \beta}=0$ при $\alpha \neq \beta$. Далее мы решаем уравнение (6) методом функций Грина на основе эквивалентных интегральных уравнений, которые последовательно учитывают вклады $\mathbf{P}^{(v)}$ с $v=$ I, II в поляризацию. Вследствие латеральной трансляционной симметрии и изотропии задачи при $\mathbf{P}^{(0)}=0$ и $\mathbf{P}^{(\mathrm{I})}$ удобно искать поля в виде $F(z, \boldsymbol{\rho})=F(z ; \kappa) \exp (i \kappa x)$, как в Приложении 1. С помощью тензорных функций Грина $\hat{g}^{(v)}\left(z, z^{\prime} ; \kappa\right)$ вида (А1.1) интегральные уравнения $v$-го приближения для поля представим в следующей форме:

$$
\begin{gathered}
\mathbf{E}^{(\mathrm{I})}-\mathbf{E}^{(0)}=\hat{g}^{(0)} \frac{\Delta \varepsilon^{(0)}}{4 \pi} \mathbf{E}^{(\mathrm{I})}=\hat{g}^{(0)} \frac{\Delta \varepsilon}{4 \pi} \mathbf{E}^{(0)}, \\
\mathbf{E}^{(\mathrm{II})}-\mathbf{E}^{(\mathrm{I})}=\hat{G}^{(\mathrm{I})} \hat{\chi}^{(0)} \mathbf{E}^{(\mathrm{II})}=\hat{G}^{(\mathrm{I})} \hat{\chi} \mathbf{E}^{(\mathrm{I})} .
\end{gathered}
$$

В общем случае знак произведения в уравнениях (7) и (8) означает интегрирование по координатам, общим для соседних функций, и суммирование по общим индексам. Вторые равенства в формулах (7) и (8) соответствуют переходу к функциям отклика $\Delta \varepsilon$ и $\hat{\chi}$ на внешнее поле. Функция Грина $\hat{g}^{(\mathrm{I})}$ определяется уравнением, которое получается из уравнения вида (7) после замены в нем $\mathbf{E}^{\left(v^{\prime}\right)} \rightarrow \hat{g}^{\left(v^{\prime}\right)}$, где $v^{\prime}=0$, I. При учете в уравнении (8) дифракции света на слое частиц функция Грина $\hat{G}^{(\mathrm{I})}$ берется в виде $(\mathrm{A} 2.2)$, а в отсутствие дифракции она заменяется на $\hat{g}^{(\mathrm{I})}$.

В дальнейшем мы рассматриваем распространение линейно поляризованных волн вдоль оси сверхрешетки $z$ в пренебрежении дифракцией волн на короткопериодных слоях наночастиц, т.е. при $\kappa=0$. Для таких волн решение уравнений (6) или (7), (8) в $v$-м приближении по $\mathbf{P}^{(v)}(v=\mathrm{I}, \mathrm{II})$ представляем в виде

$$
E^{(v)}(z)=a_{n}^{(v)} e^{i k_{b}(z-n d)}+b_{n}^{(v)} e^{-i k_{b}(z-n d)}
$$

для $z$, лежащих в области между $n$-м и $(n+1)$-м бислоями. Здесь $a_{n}^{(v)}$ и $b_{n}^{(v)}-$ постоянные, $k_{b}=\sqrt{\varepsilon_{b}} k_{0}$, а декартов индекс $\alpha=x$ или $y$, показывающий поляризацию волн, опущен. Для $n$-го BL матрицу переноса $\hat{\Lambda}$ плазмонэкситонных поляритонов определяем соотношением

$$
\left(\begin{array}{l}
a_{n} \\
b_{n}
\end{array}\right)=\hat{\Lambda}\left(\begin{array}{l}
a_{n-1} \\
b_{n-1}
\end{array}\right)
$$

где $a_{n}=a_{n}^{(\mathrm{II})}, b_{n}=b_{n}^{(\mathrm{II})}$.

Поле $\mathbf{E}^{(\mathrm{I})}$ из уравнения (7) для экситонных поляритонов, распространяющихся по нормали к квантовым ямам, выражается через коэффициенты отражения

$$
\rho_{Q W}=\frac{i \Gamma_{R}}{\omega_{0}-\omega-i \Gamma-i \Gamma_{R}}
$$

и пропускания $\tau_{Q W}=1+\rho_{Q W}$ с $\Gamma_{R}=2 \pi \Gamma_{0} k_{0} l / \sqrt{\varepsilon_{b}}$, взятым при $\omega=\omega_{0}$. Формула (11) получается при $\kappa=0$ из общих выражений (А1.3) для изолированной квантовой ямы с центром $z=0$.

Считаем, что наносфероиды занимают узлы

$$
\boldsymbol{\rho}_{\mathbf{m}}=A\left(m_{1} \mathbf{e}_{x}+m_{2} \mathbf{e}_{y}\right)
$$

квадратной решетки с периодом $A$ и номерами узлов $\mathbf{m}=\left(m_{1}, m_{2}\right)$. Каждый такой двумерно-периодический слой частиц находится на расстоянии $h \ll\left(k_{0} \sqrt{\varepsilon_{b}}\right)^{-1}$ от своей квантовой ямы. В общем случае самосогласованное решение уравнения (8) для сверхрешетки бислоев имеет вид

$$
\begin{aligned}
& E_{\beta}^{(\mathrm{II})}(z)=\delta_{\beta \alpha} E_{\alpha}^{(\mathrm{I})}(z) \\
& \quad+\sum_{\mathbf{s}} \sum_{n} e^{i \mathbf{b}_{\mathbf{s}} \boldsymbol{\rho}} G_{\beta \alpha}^{(\mathrm{I})}\left(z, z_{p, n} ; \mathbf{b}_{\mathbf{s}}\right) \chi_{\alpha \alpha}(\omega) E_{\alpha}^{(\mathrm{I})}\left(z_{p, n}\right) .
\end{aligned}
$$

Здесь проводится суммирование по номерам узлов $s_{1}$ и $s_{2}$ или векторам

$$
\mathbf{b}_{\mathbf{s}}=\frac{2 \pi}{A}\left(s_{1} \mathbf{e}_{x}+s_{2} \mathbf{e}_{y}\right)
$$

решетки, обратной по отношению к решетке (12). Волны с $\mathbf{b}_{\mathbf{s}}=0$ из выражения (13) распространяются по нормали к слоям, а волны с $\mathbf{b}_{\mathbf{s}} \neq 0$ в случае короткопериодной решетки с $A \ll k_{0}^{-1}$ соответствуют дифракции в латерально распространяющиеся волны, которые экспоненциально затухают по нормали к слою на расстояниях $\sim 1 /\left|\mathbf{b}_{\mathbf{s}}\right|$.

Выражение (13) содержит эффективную плазмонэкситонную поляризуемость $\hat{\chi}$ с компонентами $\chi_{x x}=\chi_{y y}$, относящуюся к наночастицам бислоя. В квазистатическом приближении компонента тензора $\hat{\chi}$ для моды, поляризованной вдоль $\alpha$-й полуоси сфероида, равна $[27,28]$

$$
\chi_{\alpha \alpha}(\omega)=\left(1 / \chi_{\alpha \alpha}^{(0)}-\sum_{\mathbf{m}}^{\prime} D_{\alpha \alpha}^{(\mathrm{I})}\left(z_{p, n}, z_{p, n}, \boldsymbol{\rho}_{\mathbf{m}}\right)\right)^{-1},
$$

где штрих означает исключение самодействия диполей. Полученное самосогласованное выражение (15), включающее компоненты плазмонной поляризуемости изолированной частицы $\chi_{\alpha \alpha}^{(0)}$ из (4) или (5), является 
формально точным в модели (3). Тензор $\hat{\chi}$ с компонентами (15) учитывает симметрию и резонансные свойства локализованных плазмон-экситонных мод в $\mathrm{BL}$ сверхрешетки. Функция Грина $\hat{D}^{(\mathrm{I})}=\hat{D}^{(d)}+\hat{D}^{(i)}$ с компонентами (А1.1) общего вида включает вклад $\hat{D}^{(d)}$, соответствующий прямому действию на выделенный диполь других диполей слоя, и вклад $\hat{D}^{(i)}$ их диполей изображения, порожденных экситонной поляризацией. В отсутствие квантовых ям из формулы (15) следует исключить вклад $\hat{D}^{(i)}$.

В отсутствие квантовых ям коэффициенты отражения и пропускания света слоем наночастиц с $\chi_{y y}=\chi_{x x}$ выражаются согласно (7) формулами

$$
\rho_{N P}=\frac{2 \pi i}{\sqrt{\varepsilon_{b}}} \frac{k_{0}}{A^{2}} \chi_{\alpha \alpha}, \quad \tau_{N P}=1+\rho_{N P},
$$

которые записаны здесь для слоя NP с координатой $z=0[27,28]$.

После преобразования выражения (15) с учетом формул (А2.1)-(А2.6) в пренебрежении запаздыванием $(c \rightarrow \infty)$ получаем

$$
\begin{gathered}
\chi_{\alpha \alpha}(\omega)=\left[1 / \chi_{\alpha \alpha}^{(0)}(\omega)-\sigma_{\alpha}(\omega)\right]^{-1}, \\
\sigma_{\alpha}(\omega)=-\frac{1}{2 \varepsilon_{b}} \int_{0}^{\infty} \tilde{\rho}_{Q W}^{p}(\kappa, \omega) e^{-2 \kappa h} \kappa^{2} d \kappa,
\end{gathered}
$$

где $\alpha=x, y$. Функции $\sigma_{\alpha}$ определяются ближним полем мод, имеющих латеральные компоненты волнового вектора $\kappa \sim 1 / h \gg k_{0} \sqrt{\varepsilon_{b}}$ и пространственно затухающих по $z$. Как следствие, плазмон-экситонная поляризуемость наночастицы (17), (18) выражается через коэффициент $\tilde{\rho}_{Q W}^{p},(\mathrm{~A} 2.6)$, вычисленный из (А1.3) при $\kappa \gg k_{0} \sqrt{\varepsilon_{b}}$. Это соответствует учету $p$-поляризованной компоненты поля, которая ответственна за эффекты ближнего поля [31]. Вкладом $s$-поляризованной компоненты пренебрегаем, так как он мал в отношении $\sim\left(k_{0} / \kappa\right)^{2} \ll 1$. Из выражения (17) следует, что спектр слабо затухающих кулоновских плазмонэкситонов с $\alpha$-поляризацией определяется уравнением $\operatorname{Re}\left[1 / \chi_{\alpha \alpha}^{(0)}(\omega)-\sigma_{\alpha}(\omega)\right]=0$.

При $\kappa \sim 1 / h \gg k_{0} \sqrt{\varepsilon_{b}}$ радиационное затухание квазидвумерных экситонов отсутствует: величины $\Gamma_{R}^{\alpha}$ из (А1.4) становятся мнимыми. Как показано в [16], их дисперсия по параметру $\kappa l$ и поляризация соответствующей квазистатической экситонной моды аналогичны характеристикам поверхностных плазмонов в тонких металлических пленках. В существенной области интегрирования, где $\kappa \sim 1 / h$, пренебрегаем малым членом $\Gamma_{0} \kappa l \ll \omega_{0}$ в знаменателях входящей в выражение (18) функции $\tilde{\rho}_{Q W}^{p}(\omega, \kappa)$ (см. А2.6). Тогда после вычисления интеграла в (18) получаем

$$
\sigma_{\alpha}(\omega)=\frac{1}{\varepsilon_{b} A^{3}}\left(S_{\alpha}^{(d)}+\frac{12 \pi h l}{\varepsilon_{b} A^{2}} F_{Q W}(\omega) S_{\alpha}^{(i)}\right)
$$

со спектральной функцией

$$
F_{Q W}(\omega)=\frac{\Gamma_{0}}{\omega_{0}-\omega-i \Gamma}+\frac{\Gamma_{0}}{\omega_{0}+4 \pi \Gamma_{0} / \varepsilon_{b}-\omega-i \Gamma}
$$

Эта функция относится к нерадиационным квазидвумерным экситонам квантовой ямы, которые поляризованы по осям $x$ и $z$. Малая постоянная $4 \pi \Gamma_{0} / \varepsilon_{b}<\Gamma$ в (20) служит мерой анизотропии частот этих экситонов в плоскости $x z$.

В выражение (19) входят безразмерные решеточные суммы

$$
\begin{gathered}
S_{\alpha}^{(d)}=A^{3} \sum_{\mathbf{m}(\neq 0)} \frac{3 \rho_{\mathbf{m}, \alpha}^{2}-\rho_{\mathbf{m}}^{2}}{\rho_{\mathbf{m}}^{5}}, \\
S_{\alpha}^{(i)}(h)=\left(\frac{A}{2 h}\right)^{5}-A^{5} \sum_{\mathbf{m}(\neq 0)} \frac{5 \rho_{\mathbf{m}, \alpha}^{2}-\rho_{\mathbf{m}}^{2}-4 h^{2}}{\left(\rho_{\mathbf{m}}^{2}+4 h^{2}\right)^{7 / 2}},
\end{gathered}
$$

определяющие поправки на локальное поле, действующее в узле слоя частиц. Суммы (21) обусловлены соответственно диполями, расположенными в других узлах решетки, и зарядами изображения, связанными с экситонной поляризацией квантовой ямы. Вычисление решеточных сумм описано в Приложении 2.

\section{4. Построение матрицы переноса}

Распространение поляритонов вдоль оси сверхрешетки обсудим с помощью матриц переноса из (10) для поля (9). Для изолированного BL с координатами $z_{p}=z_{0}-h / 2$ слоя частиц и $z_{e}=z_{0}+h / 2$ квантовой ямы находим коэффициенты отражения $r_{ \pm}=\rho_{ \pm} \exp \left( \pm 2 i k_{b} z_{0}\right)$ и пропускания $t_{ \pm}=\tau$ волн, распространяющихся в положительном и отрицательном направлениях оси $z$ соответственно. В этих выражениях

$$
\begin{gathered}
\rho_{+}=\rho_{Q W} e^{2 i \Delta \varphi}+\rho_{N P}\left(1+\rho_{Q W} e^{2 i \Delta \varphi}\right)^{2}, \\
\rho_{-}=\rho_{Q W}+\rho_{N P} e^{2 i \Delta \varphi}\left(1+\rho_{Q W}\right)^{2}, \\
\tau=\tau_{Q W}\left[1+\rho_{N P}\left(1+\rho_{Q W} e^{2 i \Delta \varphi}\right)\right],
\end{gathered}
$$

$k_{b}=\sqrt{\varepsilon_{b}} k_{0}$, а разность фаз $\Delta \varphi=k_{b} h$ зависит от расстояния $h$ между слоем наночастиц и квантовой ямой в BL. В терминах (22) и (23) матрица переноса в представлении (9), (10) для изолированного BL получается на основе уравнения (13) в форме

$$
\hat{\Lambda}=\frac{1}{\tau}\left(\begin{array}{cc}
\left(\tau^{2}-\rho_{+} \rho_{-}\right) e^{i k_{b} d} & \rho_{-} \\
-\rho_{+} & e^{-i k_{b} d}
\end{array}\right) .
$$

Эта матрица удовлетворяет условию унимодулярности $\operatorname{det} \hat{\Lambda}=1$.

При $\Delta \varphi \rightarrow 0$ (кулоновское взаимодействие между экситонами ямы и плазмонами слоя) получаем $\rho_{+}=\rho_{-}=\rho$ из (22) и $\tau=1+\rho$ из (23). Это позволяет непосредственно применить к сверхрешеткам, образованных бислоями металл-полупроводник, метод матриц 
переноса, развитый в работах $[22,24,32]$. Для блоховских поляритонных мод сверхрешетки в выражениях (9) и (10) полагаем $a_{n}=\lambda a_{n-1}, b_{n}=\lambda b_{n-1}$ с $\lambda=\exp ($ iqd $)$; это дает уравнение $\operatorname{det}(\hat{\Lambda}-\lambda \hat{I})=0$ с матрицей (24), имеющее решения $\lambda_{ \pm}=\exp ( \pm i q d)$. При этом квазиволновое число поляритона $q$ в сверхрешетке определяется из дисперсионного уравнения $\cos q d=\left(\Lambda_{11}+\Lambda_{22}\right) / 2$, которое при $\Delta \varphi \rightarrow 0$ в (24) принимает вид

$$
\cos q d=\cos k_{b} d+\frac{i \rho}{1+\rho} \sin k_{b} d .
$$

Следуя работам [22,24], для сверхрешетки с $N$ ячейками получаем коэффициенты отражения $r_{N}$ и пропускания $t_{N}$ света

$$
\left(r_{N}, t_{N}\right)=\frac{1}{\sin (q d) \operatorname{ctg}(q d N)-H}\left(\frac{\rho}{\tau}, \frac{\sin (q d)}{\sin (q d N)}\right)
$$

при возбуждении поляритонной моды с qd из (25) и $H=\left(\Lambda_{11}-\Lambda_{22}\right) / 2$ из $(24)$.

Особый интерес представляет режим сверхизлучения, возникающий при условии $q d=\pi[21,22]$, которое соответствует резонансной брэгговской дифракции поляритонов в сверхрешетке. Брэгговская дифракция является следствием сфазированного излучения ячейками сверхрешетки, которое сопровождается конструктивной интерференцией излученных волн. При $q \rightarrow \pi / d$ из (26) находим

$$
\left(r_{N}, t_{N}\right)=\frac{1}{N \rho-\tau}\left(N \rho,(-1)^{N-1} \tau\right)
$$

Отсюда для коэффициента отражения $R_{N}=\left|r_{N}\right|^{2}$ брэгговской сверхрешетки получаем

$$
R_{N}=\frac{N^{2}|\rho|^{2}}{|N \rho-\tau|^{2}}
$$

Из формулы (28) с $\tau=1+\rho$ следует, что $R_{N} \approx N^{2}|\rho|^{2}$, если $N|\rho| \ll 1$ (сверхизлучательный режим) и $R_{N} \rightarrow 1$ при $N \rightarrow \infty$ (режим фотонного кристалла).

\section{5. Численный анализ и обсуждение}

Обсудим результаты численного анализа оптических спектров плазмон-экситонных поляритонов, распространяющихся в ограниченных сверхрешетках. Расчеты для $\mathrm{BL}$, образованных в GaAs серебряными наночастицами и квантовыми ямами GaAs/AlGaAs, выполнены с диэлектрическими проницаемостями $\mathrm{Ag}$ [33] и GaAs [34]. Pacсматриваются дипольные плазмоны, которые в случае изолированных серебряных сфероидов (дисков) имеют поляризуемость $\chi_{x x}^{(0)}=\chi_{y y}^{(0)}$ из $(4),(5)$ в плоскости $x y$, где длина полуосей сфероидов равна $a$. При анализе отношение $\eta=b / a<1$ (длина полуоси $b$ по нормали) для сфероидов в BL выбирается так, чтобы частота плазмонного резонанса в слое частиц совпала с частотой экситонов в квантовой яме $\left(\omega_{\alpha}=\omega_{0}\right.$ при $\left.\alpha=x, y\right)$.
На рис. 2 и 3 представлены спектры отражения света (28) для металл-полупроводниковых сверхрешеток (брэгговских структур) в режиме сверхизлучения, когда выполняется условие брэгговской дифракции $q d=\pi$ на частоте резонанса $\omega_{0}=\omega_{\alpha}$. Сверхизлучение хорошо изучено для сверхрешеток с квазидвумерными экситонами при $q\left(\omega_{0}\right)=\pi / d$ или $d=\pi c /\left(\sqrt{\varepsilon_{b}} \omega_{0}\right)$ [21]. Однако для брэгговских структур с плазмонами сверхизлучение, по-видимому, не обсуждалось систематически (сравни с [35]). Для плазмонных поляритонов спектры отражения $R_{N}^{(N P)}=\left|r_{N}^{(N P)}\right|^{2}$ вычисляются по формуле (28) при условии $\rho=\bar{\rho}_{N P}$, которое следует из выражений $(16),(17)$ в отсутствие диполей изображения (квантовых ям), что отмечено чертой сверху. Спектр отражения сверхрешетки $R_{N}^{(N P)}$ при $\omega_{\alpha}=\omega_{0}$ в плазмон-поляритонном режиме сверхизлучения приведен на рис. 2,a. Для сравнения на рис. 2, $b$ показаны аналогичные экситон-поляритонные спектры отражения $R_{N}^{(Q W)}=\left|r_{N}^{(Q W)}\right|^{2}$ в сверхизлучательном режиме, вычисленные из (28) при $q\left(\omega_{0}\right) d=\pi, \rho=\rho_{Q W}$ и $\rho_{N P}=0$.

Согласно теории $[21,36,37]$, для брэгговской структуры с квантовыми ямами ширина спектра экситонного отражения света $R_{N}^{(Q W)}$ возрастает пропорционально $N^{2}$ с увеличением числа ям $N$ при $N \ll N_{c}=\Delta / \Gamma_{R}$, a затем насыщается на ширине запрещенной зоны $\Delta=\sqrt{8 \Gamma_{R} \omega_{0} / \pi}$ электромагнитного спектра сверхрешетки. В случае $N \gg N_{c}$ проявляются свойства фотонного кристалла $\left(R_{N}^{(Q W)} \rightarrow 1\right.$ в области шириной $\Delta$ с центром на резонансной частоте $\left.\omega_{0}\right)$. Переход от сверхизлучательного к фотонно-кристаллическому поведению спектра $R_{N}^{(Q W)}$ экситонных поляритонов в структуре с квантовыми ямами (выражение $(28)$ при $\rho_{N P}=0$ ) виден на рис. $2, b$ при чрезвычайно больших $N$, поскольку для экситонов $\Delta / \Gamma_{R} \sim \sqrt{\omega_{0} / \Gamma_{R}} \gg 1$.

Чтобы обсудить аналогичный переход для плазмонов, учтем (5) и представим коэффициент отражения света (16) от изолированного слоя наночастиц в виде

$$
\bar{\rho}_{N P}=\frac{i \bar{\Omega}_{\alpha}^{2}}{\bar{\omega}_{\alpha}^{2}-\omega^{2}-i \omega \gamma} .
$$

Отсутствие диполей изображения отмечено чертой сверху у величин

$$
\begin{gathered}
\bar{\omega}_{\alpha}^{2}=\left(\omega_{\alpha}^{(0)}\right)^{2}-\left(\Omega_{\alpha}^{(0)}\right)^{2} \frac{\eta}{3 \varepsilon_{b}}\left(\frac{a}{A}\right)^{3} S_{\alpha}^{(d)}, \\
\bar{\Omega}_{\alpha}^{2}=\left(\Omega_{\alpha}^{(0)}\right)^{2} \frac{2 \pi \eta}{3}\left(\frac{\bar{\omega}_{\alpha} a}{c}\right)\left(\frac{a}{A}\right)^{2} .
\end{gathered}
$$

Частоты $\omega_{\alpha}^{(0)}$ и $\Omega_{\alpha}^{(0)}$ определяются формулой (5), а сумма $S_{\alpha}^{(d)}=4.51-$ формулой $(21)$. Учитывая аналогии $\omega_{0} \rightarrow \bar{\omega}_{\alpha}$ и $\Gamma_{R} \rightarrow \bar{\Omega}_{\alpha} \sim \bar{\omega}_{\alpha}$ между формулами (11) и (29), для плазмонных поляритонов в брэгговской структуре со слоями наночастиц получаем оценку ширины запрещенной зоны $\sim \omega_{\alpha}$ и $N_{c} \sim 1$. Это означает, что в сверхрешетке из слоев наночастиц плазмон-поляритонный 

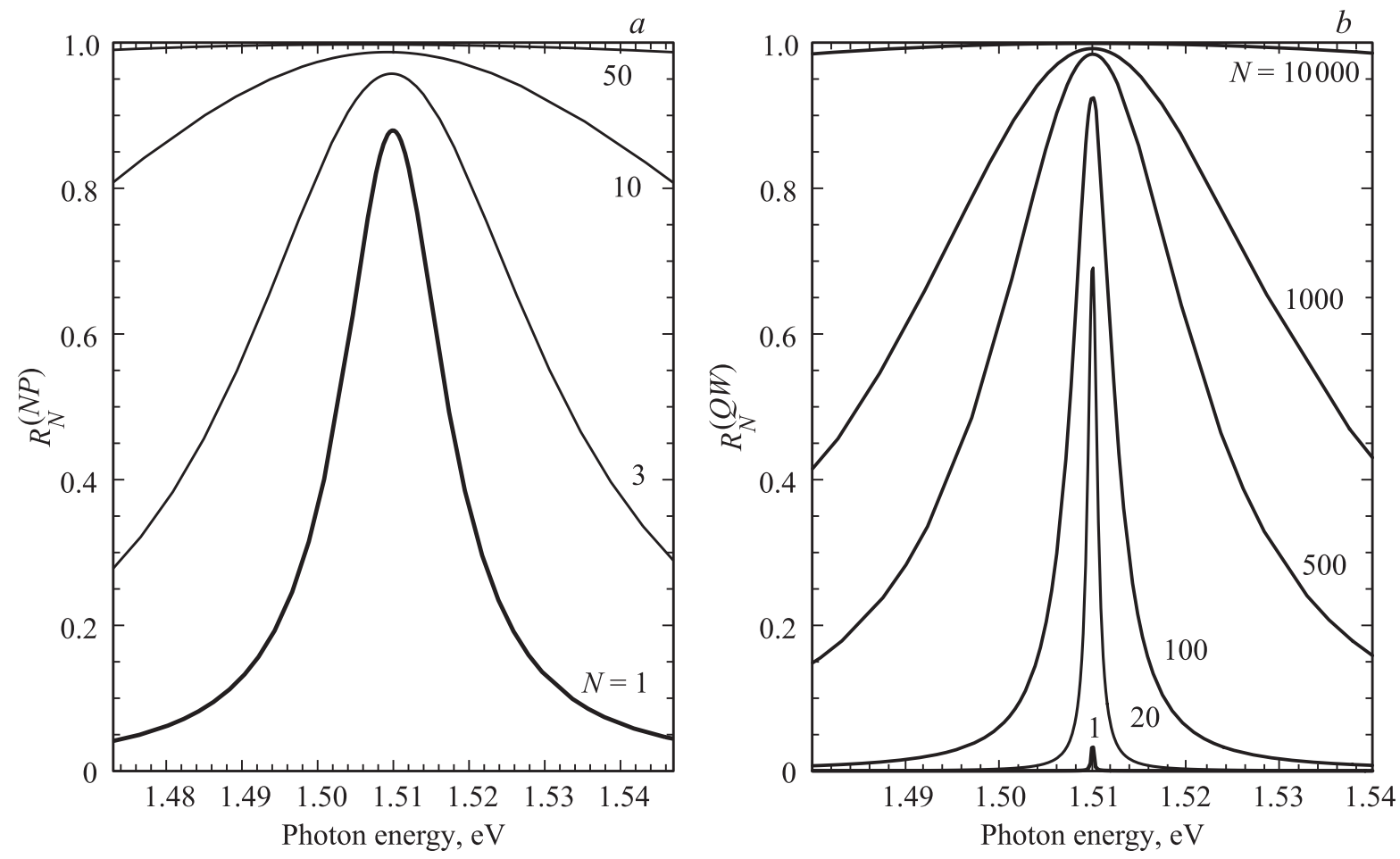

Рис. 2. Спектры отражения в режиме сверхизлучения $(q d=\pi)$ брэгговских структур $(a)$ с $N$ слоями сфероидов (дисков) $\mathrm{Ag}-R_{N}^{(N P)}$, (b) с квантовыми ямами $\mathrm{GaAs} / \mathrm{AlGaAs}-R_{N}^{(Q W)}$. Вычислено при $\omega_{\alpha}=\omega_{0}$, где $\omega_{\alpha}-$ частота плазмонов, поляризованных по осям $\alpha=x, y$ сфероидов с длинами полуосей $a_{x}=a_{y}=a=8 \mathrm{~nm}$, отношением $b / a=0.596$ в слоях с периодом $A=25 \mathrm{~nm}$ и $h=9 \mathrm{~nm}$. Принято $\hbar \omega_{0}=1.51 \mathrm{eV}, \hbar \Gamma=0.1 \mathrm{meV}, \hbar \Gamma_{R}=0.025 \mathrm{meV}\left(\hbar \Gamma_{0}=0.23 \mathrm{meV}\right)$ для экситонов, $\varepsilon_{b}=12$ для GaAs и $l=8 \mathrm{~nm}$ для квантовых ям. Использована диэлектрическая проницаемость $\mathrm{Ag}$ из [33], аппроксимированная функцией $\varepsilon(\omega)=\varepsilon_{\infty}-\omega_{p}^{2} /\left(\omega^{2}+i \omega \gamma\right)$ с $\hbar \omega_{p}=9.45 \mathrm{eV}, \hbar \gamma=0.02 \mathrm{eV}$ и $\varepsilon_{\infty}=4.7$.

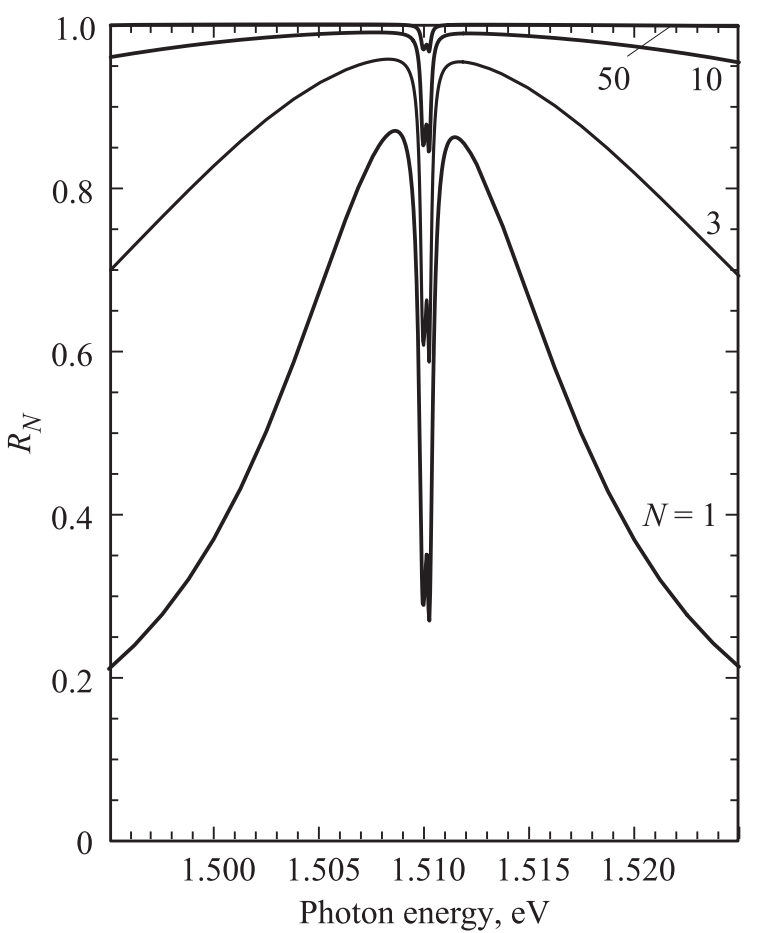

Рис. 3. Спектры плазмон-экситонного отражения $R_{N}$ в режиме сверхизлучения от сверхрешеток с $N$ бислоями, образованными упорядоченным слоем наночастиц $\mathrm{Ag}$ и квантовой ямой GaAs/AlGaAs. Параметры те же, что на рис. 2. сверхизлучательный режим возможен только при сравнительно малых числах слоев $N$, а ширина спектра отражения при переходе к фотонно-кристаллическому режиму оказывается сравнимой с частотой плазмонов $\omega_{\alpha}$ в частицах. Следует заметить также, что по сравнению с идеальной картиной перехода от одного режима отражения к другому в случае экситонов картина перехода в случае плазмонов существенно усложняется из-за значительного поглощения света (при энергии фотонов выше $4 \mathrm{eV}$ для $\mathrm{Ag}$ [27]).

На рис. 3 для сверхрешеток с разным числом $N$ бислоев показаны вычисленные из (28) спектры $R_{N}=\left|r_{N}\right|^{2}$ плазмон-экситонного отражения света в режиме сверхизлучения. Спектр отражения, показанный на рис. 3 кривой 1 для $N=1$, соответствует исследованным в работе [16] спектрам отражения и поляризуемости $\chi_{\alpha \alpha}(\omega)$ BL при наличии плазмон-экситонного взаимодействия через ближнее поле. С другой стороны, показанные на рис. 3 спектры отражения $R_{N}$ брэгговскими структурами с разными $N$ в целом близки к спектрам плазмон-поляритонного сверхизлучения, представленным на рис. $2, a$. В области резонанса $\omega_{\alpha}=\omega_{0}$ в спектрах $R_{N}$ (рис. 3) имеется узкий провал изза плазмон-экситонного расщепления Раби, но эта особенность быстро уменьшается при увеличении числа ячеек $N$ сверхрешетки. 


\section{6. Заключение}

В работе представлена теория плазмон-экситонных поляритонов в сверхрешетках, образованных па́рами близко расположенных квантовых ям и слоев металлических наночастиц. Резонансное взаимодействие квазидвумерных экситонов с локальными плазмонами исследовано применительно к квантовым ямам GaAs/AlGaAs и слоям частиц благородного металла. Показано, что смешанные плазмон-экситонные поляритоны в сверхрешетке имеют широкий спектр, обусловленный плазмоном, с узким спектральным провалом в области экситонного резонанса. Режим сверхизлучения при резонансной брэгговской дифракции поляритонов исследован для сверхрешеток с экситонами, плазмонами и смешанными плазмон-экситонными модами. Установлено, что переход от сверхизлучательного к фотонно-кристаллическому режиму (полному отражению) при увеличении числа ячеек в сверхрешетке с плазмонами происходит значительно быстрее, чем в сверхрешетке с экситонами. В случае смешанных плазмон-экситонных мод такой переход определяется плазмонами, причем спектральный провал, связанный с плазмон-экситонным расщеплением Раби, быстро уменьшается при увеличении числа ячеек сверхрешетки.

Автор благодарен А.Н. Поддубному за обсуждение результатов работы и замечания.

\section{Приложение 1. \\ Решение задачи для квантовой ямы}

Уравнения вида (7) дают поле и функции Грина

$$
\left\{E_{\alpha}^{(\mathrm{I})}(z, x), g_{\alpha \beta}^{(\mathrm{I})}\left(z, z^{\prime}, x\right)\right\}=\left\{E_{\alpha}^{(\mathrm{I})}(z ; \kappa), g_{\alpha \beta}^{(\mathrm{I})}\left(z, z^{\prime}, \kappa\right)\right\} e^{i \kappa x}
$$

с $\boldsymbol{\kappa}=\kappa \mathbf{e}_{x}$. Вне квантовой ямы с центром $z=z_{e}$ волна с амплитудой $E_{\alpha}^{\text {inc }}$ и $\kappa \neq 0$ возбуждает поле

$$
\begin{aligned}
\frac{E_{\alpha}^{(\mathrm{I})}(z, \kappa)}{E_{\alpha}^{\text {inc }}}= & \left(e^{i k_{1} z}+\rho_{Q W}^{\lambda} e^{2 i k_{1} z_{e}} e^{-i k_{1} z}\right) \vartheta\left(z_{e}-z\right) \\
& +\tau_{Q W}^{\lambda} e^{i k_{1} z} \vartheta\left(z-z_{e}\right) .
\end{aligned}
$$

Здесь $\vartheta(z)$ - единичная ступенчатая функция, $\alpha=x$ для линейной поляризации $\lambda=p$ и $\alpha=y$ для $\lambda=s$, a $k_{1}^{2}=\varepsilon_{b} k_{0}^{2}-\kappa^{2}>0$. В (А1.2) входят коэффициенты экситонного отражения света

$$
\begin{gathered}
\rho_{Q W}^{p}=\frac{i \Gamma_{R}^{x}}{\omega_{0}^{x}-\omega-i \Gamma-i \Gamma_{R}^{x}}-\frac{i \Gamma_{R}^{z}}{\omega_{0}^{z}-\omega-i \Gamma-i \Gamma_{R}^{z}}, \\
\rho_{Q W}^{s}=\frac{i \Gamma_{R}^{y}}{\omega_{0}^{y}-\omega-i \Gamma-i \Gamma_{R}^{y}}
\end{gathered}
$$

квантовой ямой. В формулах (А1.3) $\omega_{0}^{\alpha}=\omega_{0}+\Delta \omega_{0}^{\alpha}$,

$$
\left.\begin{array}{c}
\left(\Delta \omega_{0}^{x}, \Delta \omega_{0}^{z} ; \Delta \omega_{0}^{y}\right)=\Gamma_{0} w(0,2 ; 0), \\
\left(\Gamma_{R}^{x}, \Gamma_{R}^{z} ; \Gamma_{R}^{y}\right)=\Gamma_{0} w k_{1} l\left(1, \kappa^{2} / k_{1}^{2} ; \varepsilon_{b} k_{0}^{2} / k_{1}^{2}\right)
\end{array}\right\},
$$

где $w=2 \pi / \varepsilon_{b}$.
Для $p$-поляризованных волн компоненты функции Грина в (А1.1) равны

$$
\begin{aligned}
g_{x x}^{(\mathrm{I})}\left(z, z^{\prime} ; \kappa\right)= & \frac{2 \pi i k_{1}}{\varepsilon_{b}}\left[e^{i k_{1}\left|z-z^{\prime}\right|}+\rho_{Q W}^{p} e^{2 i k_{1} z_{e}} e^{-i k_{1}\left(z+z^{\prime}\right)}\right] \\
g_{z x}^{(\mathrm{I})}\left(z, z^{\prime} ; \kappa\right)= & -\frac{2 \pi i \kappa}{\varepsilon_{b}}\left[e^{i k_{1}\left|z-z^{\prime}\right|} \operatorname{sgn}\left(z-z^{\prime}\right)\right. \\
& \left.-\rho_{Q W}^{p} e^{2 i k_{1} z_{e}} e^{-i k_{1}\left(z+z^{\prime}\right)}\right]
\end{aligned}
$$

и т. д., где $z, z^{\prime}<z_{0}$, а $\rho_{Q W}^{p}(\kappa)$ дается формулами (А1.3) и (А1.4). Для $s$-поляризованных волн

$$
g_{y y}^{(\mathrm{I})}\left(z, z^{\prime} ; \kappa\right)=\frac{2 \pi i k_{0}^{2}}{k_{1}}\left[e^{i k_{1}\left|z-z^{\prime}\right|}+\rho_{Q W}^{s} e^{2 i k_{1} z_{e}} e^{-i k_{1}\left(z+z^{\prime}\right)}\right]
$$

с $\rho_{Q W}^{s}$ из (А1.3) и (А1.4). Отсюда следует, что $\left|g_{y y}^{(\mathrm{I})}\right| /\left|g_{x x}^{(\mathrm{I})}\right| \sim\left(k_{0} / \kappa\right)^{2} \ll 1$ при $\kappa \gg k_{0}$.

\section{Приложение 2.}

\section{Вычисление решеточных сумм}

Для анализа ближнеполевых эффектов используется преобразование Фурье

$$
\begin{gathered}
D_{\alpha \beta}^{(\mathrm{I})}\left(z, z^{\prime}, \boldsymbol{\rho}\right)=\int \frac{d^{2} \kappa}{(2 \pi)^{2}} e^{i \kappa \boldsymbol{\rho}} G_{\alpha \beta}^{(\mathrm{I})}\left(z, z^{\prime} ; \boldsymbol{\kappa}\right), \\
G_{\alpha \beta}^{(\mathrm{I})}\left(z, z^{\prime} ; \boldsymbol{\kappa}\right)=\sum_{\mu, \nu} S_{\alpha \mu}(\phi) g_{\mu \nu}^{(\mathrm{I})}\left(z, z^{\prime} ; \kappa\right) S_{\beta \nu}(\phi)
\end{gathered}
$$

функции Грина $\hat{g}^{(\mathrm{I})}$, вычисленной в представлении (А1.1). Здесь $S_{x x}=S_{y y}=\kappa_{x} / \kappa=\cos \phi, \quad-S_{x y}=$ $=S_{y x}=\kappa_{y} / \kappa=\sin \phi, S_{z z}=1, \phi-$ угол между вектором $\boldsymbol{\kappa}$ и осью $x$. После интегрирования по углу $\phi$ с учетом (А2.2) выражение (А2.1) в квазистатическом приближении принимает вид [31]

$$
\begin{aligned}
& \tilde{\tilde{D}}^{(\mathrm{I})}\left(z, z^{\prime}, \boldsymbol{\rho}\right)=\int_{0}^{\infty} \frac{\kappa d \kappa}{4 \pi} \\
& \times\left(\begin{array}{ccc}
\tilde{g}_{x x}^{(\mathrm{I})}\left(J_{0}-J_{2} \cos 2 \varphi\right) & -\tilde{g}_{x x}^{(\mathrm{I})} J_{2} \sin 2 \varphi & 2 i \tilde{g}_{x z}^{(\mathrm{I})} J_{1} \cos \varphi \\
-\tilde{g}_{x x}^{(\mathrm{I})} J_{2} \sin 2 \varphi & \tilde{g}_{x x}^{(\mathrm{I})}\left(J_{0}+J_{2} \cos 2 \varphi\right) & 2 i \tilde{g}_{x z}^{(\mathrm{I})} J_{1} \sin \varphi \\
2 i \tilde{g}_{z x}^{(\mathrm{I})} J_{1} \cos \varphi & 2 i \tilde{g}_{z x}^{(\mathrm{I})} J_{1} \sin \varphi & 2 \tilde{g}_{z z}^{(\mathrm{I})} J_{0}
\end{array}\right) .
\end{aligned}
$$

Здесь $\varphi-$ угол между радиус-вектором $\rho$ и осью $x$, $J_{m} \equiv J_{m}(\kappa \rho)-$ функция Бесселя аргумента $\kappa \rho$, а $\tilde{D}_{\alpha \beta}^{(\mathrm{I})}$ и $\tilde{g}_{\alpha \beta}^{(\mathrm{I})}$ обозначают компоненты $D_{\alpha \beta}^{(\mathrm{I})}$ из $(\mathrm{A} 2.1)$ и $g_{\alpha \beta}^{(\mathrm{I})}$ из $(\mathrm{A} 1.5)$, вычисленные при $k_{0} / \kappa \rightarrow 0$. Компонента $\tilde{g}_{y y}^{(\mathrm{I})}$ из формулы (А2.3) исключена, так как в квазистатическом приближении (при $\kappa \gg k_{0}$ ) она мала по параметру $\sim\left(k_{0} / \kappa\right)^{2} \ll 1$ по сравнению с другими компонентами $g_{\alpha \beta}^{(\mathrm{I})}$, что следует из (А1.5) и (А1.6). 
При подстановке в выражение (А2.3) функции Грина $\tilde{g}_{\alpha \beta}^{(\mathrm{I})}=\tilde{g}_{\alpha \beta}^{(d)}+\tilde{g}_{\alpha \beta}^{(i)}$ с $k_{1} \approx i \kappa$ возникают интегралы

$$
F_{n, m}(\xi, \rho)=\int_{0}^{\infty} d \kappa \kappa^{n-1} J_{m}(\kappa \rho) e^{-\kappa \xi}
$$

С учетом вклада в функцию Грина $\tilde{g}_{\alpha \beta}^{(d)}=C_{d} \kappa e^{-\kappa \xi}$, соответствующего отсутствию квантовой ямы $\left(\rho_{Q W}^{p}=0\right)$, получаем

$$
\begin{aligned}
F_{3, m}(\xi, \rho)= & \frac{1}{\left(\xi^{2}+\rho^{2}\right)^{5 / 2}} \\
& \times\left[\left(2 \xi^{2}-\rho^{2}\right) \delta_{m, 0}+3 \xi \rho \delta_{m, 1}+3 \rho^{2} \delta_{m, 2}\right] .
\end{aligned}
$$

Вклад $\tilde{g}_{\alpha \beta}^{(i)}=C_{i} \kappa^{2} e^{-\kappa \xi}$, связанный с поляризацией квантовой ямы (с зарядами изображения), содержит величину

$$
\begin{aligned}
\tilde{\rho}_{Q W}^{p}=-w \kappa l & \frac{\Gamma_{0}}{\omega_{0}+w \Gamma_{0} \kappa l-\omega-i \Gamma} \\
& \left.+\frac{\Gamma_{0}}{\omega_{0}+w \Gamma_{0}(2-\kappa l)-\omega-i \Gamma}\right)
\end{aligned}
$$

c $w=2 \pi / \varepsilon_{b}$. Пренебрегая в знаменателях (А2.6) членами с $\kappa l \ll 1$, находим

$$
\begin{aligned}
F_{4, m}(\xi, \rho)= & \frac{1}{\left(\xi^{2}+\rho^{2}\right)^{7 / 2}}\left[3 \xi\left(2 \xi^{2}-3 \rho^{2}\right) \delta_{m, 0}\right. \\
& \left.+3 \rho\left(4 \xi^{2}-\rho^{2}\right) \delta_{m, 1}+15 \xi \rho^{2} \delta_{m, 2}\right] .
\end{aligned}
$$

Подставим в (А2.3) выражения (А2.5) и (А2.7), взятые при $\boldsymbol{\rho}=\boldsymbol{\rho}_{\mathbf{m}}$ из $(12)$, и вычислим суммы $\sum_{\mathbf{m}}^{\prime} D_{\alpha \beta}^{(\mathrm{I})}$ по номерам узлов $m_{1}$ и $m_{2}$. При этом ненулевыми оказываются только суммы $\sum_{\mathbf{m}}^{\prime} D_{\alpha \alpha}^{(\mathrm{I})}$ диагональных членов с $\alpha=\beta$, четных по $m_{1}$ и $m_{2}$.

\section{Список литературы}

[1] E.L. Ivchenko. Optical spectroscopy of semiconductor nanostructures. Alpha Science International, Ltd. (2005). 315 p.

[2] В.В. Климов. Наноплазмоника. Физматлит, М. (2010). $489 \mathrm{c}$.

[3] A.A. Toropov, T.V. Shubina. Plasmonic Effects in MetalSemiconductor Structures. Oxford Univ. Press (2015). 361 p.

[4] M. Achermann. J. Phys. Chem. Lett. 1, 2837 (2010).

[5] P. Vasa, R. Pomraenke, S. Schwieger, Yu.I. Mazur, Vas. Kunets, P. Srinivasan, E. Johnson, J.E. Kihm, D.S. Kim, E. Runge, G. Salamo, C. Lienau. Phys. Rev. Lett. 101, 116801 (2008).

[6] J. Bellessa, C. Bonnand, J.C. Plenet, J. Mugnier. Phys. Rev. Lett. 93, 036404 (2004).

[7] Y. Sugawara, T.A. Kelf, J.J. Baumberg, M.E Abdelsalam, P.N. Bartlett. Phys. Rev. Lett. 97, 266808 (2006).
[8] S. Balci. Opt. Lett. 38, 4498 (2013).

[9] B.G. DeLacy, O.D. Miller, C.W. Hsu, Z. Zander, S. Lacey, R. Yagloski, A.W. Fountain, E. Valdes, E. Anquillare, M. Soljacic, S.G. Johnson, J.D. Joannopoulos. Nano Lett. 15, 2588 (2015).

[10] B.J. Lawrie, K.-W. Kim, D.P. Norton, R.F. Haglund Jr. Nano Lett. 12, 6152 (2012).

[11] W. Zhang, A.O. Govorov, G.W. Bryant. Phys. Rev. Lett. 97, 146804 (2006).

[12] Y. Fedutik, V.V. Temnov, O. Schops, U. Woggon, M.V. Artemyev. Phys. Rev. Lett. 99, 136802 (2007).

[13] A.O. Govorov, G.W. Bryant, W. Zhang, T. Skeini, J. Lee, N.A. Kotov, J.M. Slocik, R.R. Naik. Nano Lett. 6, 984 (2006).

[14] A.A. Toropov, T.V. Shubina, K.G. Belyaev, S.V. Ivanov, P.S. Kop'ev, Y. Ogawa, F. Minami. Phys. Rev. B 84, 085323 (2011).

[15] A. Neogi, C.-W. Lee, H.O. Everitt, T. Kuroda, A. Tackeuchi, E. Yablonovitch. Phys. Rev. B 66, 153305 (2002).

[16] В.А. Кособукин. ФТТ 57, 1413 (2015).

[17] V.A. Kosobukin. Solid State Commun. 228, 43 (2016).

[18] W. Niu, L.A. Ibbotson, D. Leipold, E. Runge, G.V. Prakash, J.J. Baumberg. Phys. Rev. B 91, 161303(R) (2015).

[19] S. Campione, A. Benz, J.F. Klem, M.B. Sinclair, I. Brener, F. Capolino. Phys. Rev. B 89, 165133 (2014).

[20] M. Gehl, S. Zandbergen, R. Gibson, M. Béchu, N. Nader, J. Hendrickson, J. Sears, P. Keiffer, M. Wegener, G. Khitrova. J. Opt. 16, 114016 (2014).

[21] Е.Л. Ивченко, А.Н. Поддубный. ФТТ 55, 833 (2013).

[22] Е.Л. Ивченко, А.И. Несвижский, С. Йорда. ФТТ 36, 2118 (1994).

[23] E.L. Ivchenko, M.M. Voronov, M.V. Erementchouk, L.I. Deych, A.A. Lisyansky. Phys. Rev. B 70, 195106 (2004).

[24] В.А. Кособукин, М.М. Моисеева. ФТТ 37, 3694 (1995).

[25] L.C. Andreani, F. Bassani. Phys. Rev. B 41, 7536 (1990).

[26] Е.Л. Ивченко. ФТТ 33, 2388 (1991).

[27] В.А. Кособукин. ФТТ 54, 2340 (2012).

[28] В.А. Кособукин, А.В. Коротченков. ФТТ 58, 2446 (2016).

[29] Л.Д. Ландау, Е.М. Лифшиц. Электродинамика сплошных сред. Наука, М. (1982). 620 с.

[30] V.A. Kosobukin. Phys. Lett. A 160, 584 (1991).

[31] В.А. Кособукин. ФТТ 36, 3015 (1994).

[32] В.А. Кособукин. ФТТ 34, 3107 (1992).

[33] P.B. Johnson, R.W. Christy. Phys. Rev. B 6, 4370 (1972).

[34] D.E. Aspnes, A.A. Studna. Phys. Rev. B 27, 985 (1983).

[35] R. Taubert, D. Dregely, T. Stroucken, A. Christ, H. Giessen. Nature Commun. 3:691 doi: 10.1038/ncomms1694 (2012).

[36] E.L. Ivchenko, M. Willander. Phys. Status Solidi B 215, 199 (1999).

[37] L.I. Deych, A.A. Lisyansky. Phys. Rev. B 62, 4242 (2000). 